\title{
Research Paper \\ The Effect of Computer-based Cognitive Rehabilitation Therapy on Difficulties in Emotion-regulation among Students With Attention Deficit Hyperactivity Disorder
}

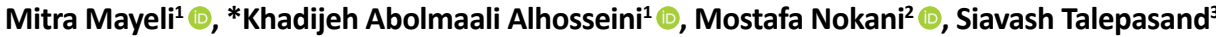 \\ 1. Department of Psychology, Faculty of Psychology and Social Sciences, Roudehen Branch, Islamic Azad University, Roudehen, Iran. \\ 2. Depratment of Psychiatry, School of Medicine, Arak University of Medical Sciences, Arak, Iran. \\ 3. Department of Educational Psychology, Faculty of Psychology and Educational Sciences, Semnan University, Semnan, Iran
}

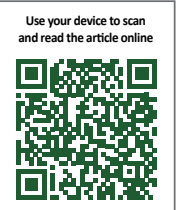

Crtation: Mayeli M, Abolmaali Alhosseini Kh, Nokani M, Talepasand S. [The Effect of Computer-based Cognitive Rehabilitation Therapy on Difficulties in Emotion-regulation among Students With Attention Deficit Hyperactivity Disorder (Persian)]. Complementary Medicine Journal. 2020; 10(3):230-243. https://doi.org/10.32598/cmja.10.3.1005.1

https://doi.org/10.32598/cmja.10.3.1005.1

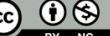

Article Info:

Received: 28 Jun 2020

Accepted: 26 Sep 2020

Available Online: 01 Oct 2020

Key words:

Attention deficit hyperactivity disorder, Computer-based cognitive rehabilitation, Difficulties in emotion regulation

\section{ABSTRACT}

ObjectiveThe aim of the present study is to investigate the effect of computer-based cognitive rehabilitation therapy on difficulties in emotional regulation among students with Attention Deficit Hyperactivity Disorder (ADHD).

Methods Participants were 24 students with ADHD (12 boys and 12 girls) studying in 6-9 ${ }^{\text {th }}$ grades during 2018-19 at schools located in District 7 of Tehran, Iran. They were selected using a purposive sampling method and based on inclusion/exclusion criteria and then randomly assigned into experimental and control groups. Data collection tool was the Difficulties in Emotion Regulation Scale (DERS) completed at pre-test, post-test, and follow-up phases. Experimental group received computer-based cognitive rehabilitation therapy for 10 weeks (20 sessions, two sessions per week, each for 30 minutes). Data were analyzed using repeated measure ANOVA in SPSS V. 26 software.

Results All subscales of DERS were significantly improved after intervention which had a medium-to-large effect size.

Conclusion Computer-based cognitive rehabilitation therapy can help treat difficulties in emotional regulation and improve performance of students with ADHD.

\section{Extended Abstract}

\section{Introduction}

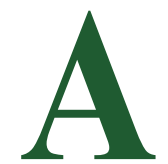

ttention Deficit Hyperactivity Disorder (ADHD) is a persistent pattern of inattention and/or hyperactive-impulsive behaviors. For its diagnosis, some symptoms must appear before the age of 12 years, although many cases are diagnosed years after the onset of symptoms. The disorder must be present in at least two fields, and the individual's performance must be impaired in social, educational, or occupational settings, depending on the rate of growth. The symptoms do not occur exclusively during the course of a pervasive developmental disorder, schizophrenia, or other psychotic disorders, and are not better accounted for by another mental disorder (e.g. mood disorder, anxiety disorder, dissociative disorder, or a personality disorder) $[1,2]$. The relationship between ADHD diagnosis education and its spread is evident. Diagnostic criteria for ADHD are influenced by factors related to education

* Corresponding Author:

Khadijeh Abolmaali Alhosseini, PhD.

Address: Department of Psychology, Faculty of Psychology and Social Sciences, Roudehen Branch, Islamic Azad University, Roudehen, Iran. Tel: +98 (912) 6265804

E-mail: sama.abolmaali@gmail.com 
[3]. In fact, in the early conceptualization of ADHD as a minimal brain damage, emotion dysregulation is the core symptom along with its main symptoms [5]. After publication of the fifth edition of Diagnostic and Statistical Manual of Mental Disorders, emotional symptoms became manifestations of ADHD dependent on the diagnostic criteria [6].

Attention is an important component of emotional regulation [12]. Automatic biases in attention and difficulties in disengaging attention may be the important attention mechanisms in emotional regulation [13, 14]. Inefficient attentional biases (towards negative emotional information) may lead to unconscious, automatic and inflexible negative evaluations of a situation [12]. Attentional bias can cause a person to pay attention to the negative aspects of a situation. This causes the person to respond negatively to the situation, which makes it more difficult to activate positive or neutral assessments of the situation and regulate negative emotions. The ability to apply reassessment may depend on a person's ability to overcome bias and self-assessment [14]. More bias in attention may increase the likelihood of distraction from the negative aspects of the situation, and the person may pay more attention to the more positive or neutral aspects of the situation, and may increase the person's ability to positively assess the situation [15]. Due to the role of attention in emotion regulation, attention training may be a valuable intervention for improving emotion regulation [16]. In a review study by Wadlinger and Scakowitz, [12] it was reported that correction of attentional bias can directly modify the processes that are important in regulating attention [17].

Rehabilitation of cognitive deficits has attracted the attention of neuropsychologists for a long time. Interventions to improve or reduce cognitive deficits generally fall into three categories: A. Environmental interventions that provide situational support for impaired abilities; B. Interventions aimed at compensating for existing deficiencies; and C. Direct interventions aimed at improving the underlying cognitive processes and eliminating/reducing the defect itself. Direct interventions assume that attention al abilities are improved by providing structured opportunities to practice different aspects of attention. Treatment involves repeated exercises with a set of tasks that require attention at different levels. It is hypothesized that repeated activation and continuous stimulation of attention systems causes a change in cognitive capacity, which seems to indicate an underlying change in neuronal activity [18].

\section{Materials and Methods}

This is an experimental study with a pre-test, post-test, follow-up design with a control group. The study population consists of all students diagnosed with ADHD in 6-9 $9^{\text {th }}$ grades in schools located in District 7 of Tehran, Iran during 2017-2018. Of these, 24 (12 boys and 12 girls) were selected using a purposive sampling method and based on the entry and exit criteria. They were then randomly divided into experimental and control groups. Symptoms of ADHD were first diagnosed in school by a school counselor expert in psychology, and then a psychiatrist confirmed ADHD diagnosis. Entry criteria were: Diagnosis of ADHD and willingness to participate in the study, while the exit criteria were: organic disorder, psychotic disorder, mental retardation, and receiving medication or any concomitant psychological intervention. The computer-based cognitive rehabilitation therapy was presented at 20 sessions, each for 30 minutes.

\section{Results}

The results of within-group comparison showed the significant effects of study variables on both study groups over time. All of the study variables were significantly changed from the pre-test to the follow-up phases. The results of pairwise comparison in the experimental group showed a significant difference in the Difficulties in Emotion Regulation Scale (DERS) dimensions between pre-test and post-test scores $(\mathrm{t}=10.43$, $\mathrm{P}=0.01$ ), between pre-test and follow-up scores $(\mathrm{t}=10.22, \mathrm{P}=0.01)$, and between post-test and follow-up scores $(\mathrm{t}=-5.19, \mathrm{P}=0.01)$. There was no significant difference between the measurement phases in the control group $(\mathrm{P}>0.05)$. Regarding between-group comparison, results showed a significant difference in the post-test and follow-up scores of all DERS dimensions between the experimental and control groups which confirms the main hypothesis of our study.

According to the ETA squared value which shows the effect size of the computer-based cognitive rehabilitation therapy, this intervention had a small effect on DERS dimensions of limited access to emotion regulation strategies $\left(\eta^{2}=0.21\right)$ and lack of emotional clarity $\left(\eta^{2}=0.27\right)$; a medium effect on dimensions of nonacceptance of emotional responses $\left(\eta^{2}=0.43\right)$, difficulty engaging in goal-directed behavior $\left(\eta^{2}=34\right)$, and lack of emotional awareness $\left(\eta^{2}=0.41\right)$; and a large effect on the dimension of impulse control difficulties $\left(\eta^{2}=0.55\right)$, and the overall DERS score $\left(\eta^{2}=0.84\right)$. 


\section{Conclusion}

The present study aimed to improve the difficulty in emotional regulation of students with ADHD. Interventions related to ADHD usually focus on its cognitive neurological symptoms or secondary behavioral problems [55]. Although psychological interventions for ADHD vary in scope, they all tend to focus on correcting destructive or distracting behaviors. Several medications have been proposed for treating the emotional and behavioral difficulties of students with ADHD due to the high difficulty of emotion regulation and associated emotional/behavioral disorders. Almost half of the children in studies by Winterstein [57] and Rosen et al. [37] received one or more medications before the intervention. The concern may be because of long-term use of psychotropic drugs and their potential side effects including loss of appetite, sleep disorders, irritability, aggression, anxiety, tics, and seizures [28].

Although there is evidence that behavioral interventions are effective in improving the performance of children with ADHD in the presence of concomitant disorders [58], the main focus of these behavioral therapies is to reduce the disorder and increase behavioral flexibility rather than to improve difficulty in emotional regulation. The intervention in the present study was able to directly improve the emotional regulation abilities of children with ADHD. Therefore, the use of non-pharmacological methods such as computer-based cognitive rehabilitation therapy, which has been reported to have no side effects on children, is need for the treatment of ADHD.

\section{Ethical Considerations}

Compliance with ethical guidelines

The APA, IRPCO, and Helsinki Ethical Codes was considered in the this study.

\section{Funding}

This study was extracted from the PhD. dissertation of first author approved by Islamic Azad University of Roudehen Branch.

\section{Authors' contributions}

All authors contributed equally in preparing all parts of the research.

\section{Conflicts of interest}

The authors declare no conflict of interest 


\title{
اثربخشى بازتوانى شناختى با استفاده از رايانه بر دشوارىهاى تنظيم هيجانى دانش آموزان مبتلا به اختلال بيشى بازتوانى كمبود توجني
}

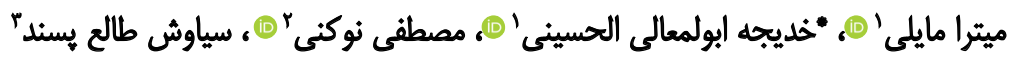

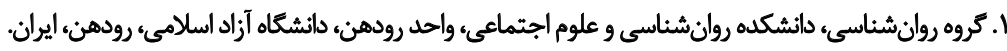

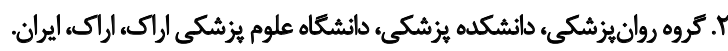

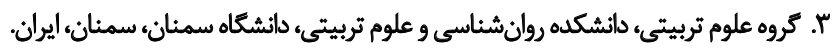

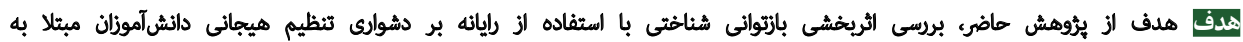
اخثلال بيش فيشالى كمبود توجه بودي.

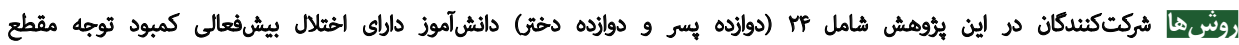

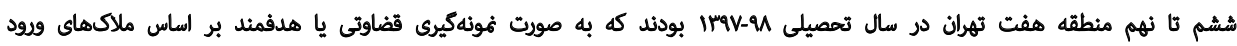

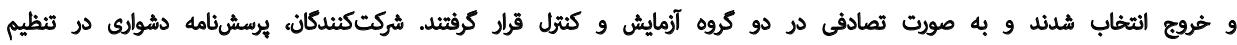

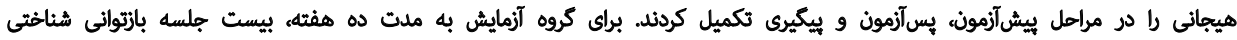

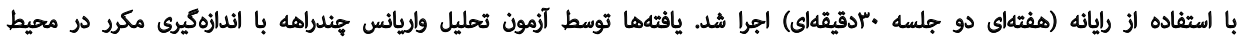
نرمافزار SPSS نسخغه وع مورد تعليل قرار كرفئند.

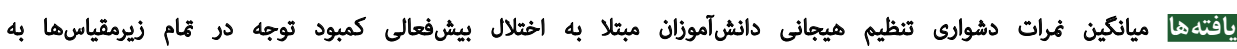

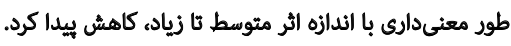

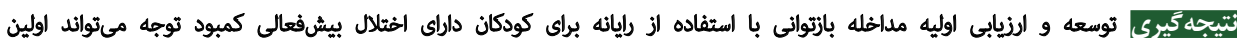

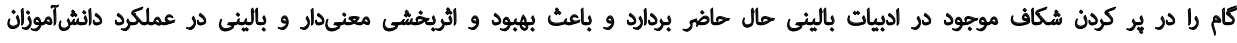

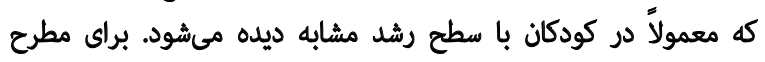

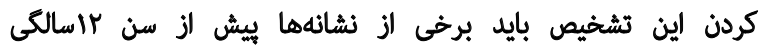

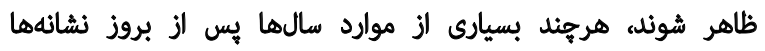

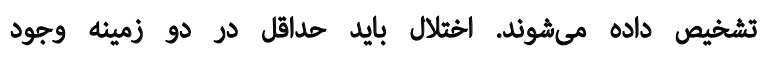

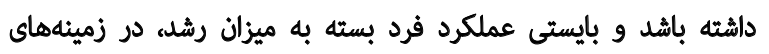

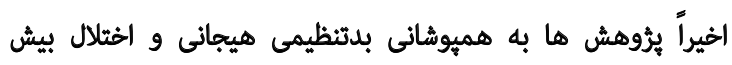

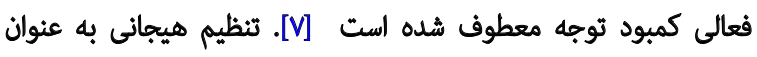

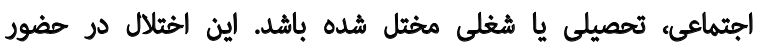

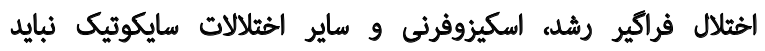
مطرح شود و نيز يك اختلال روانى ديكر توجيه بهترى برائ آن آن 1. Attention Deficit Hyperactivity Disorder (ADHD)

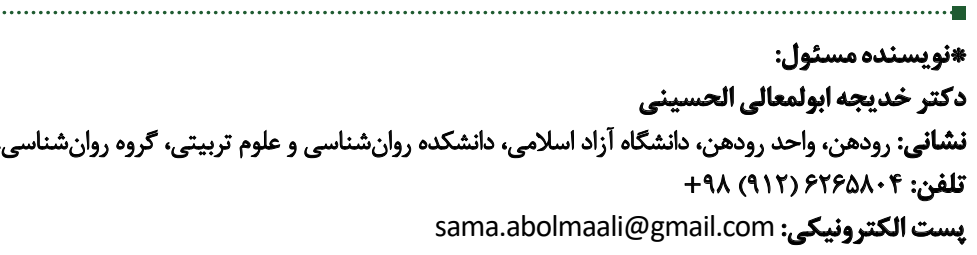


مرورى، وادلينكر و ايساكوويتز [II] به اين نتيجه رسيدند كه اصلاح

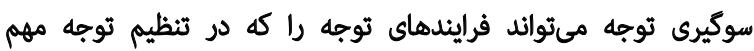

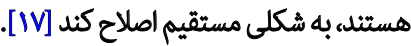

بازتوانى نقصهاى شناختى به مدت طولانى ذهن من متخصصان

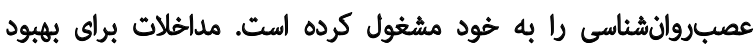

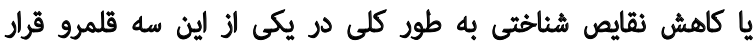

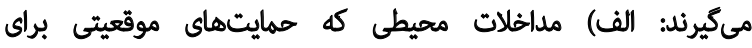

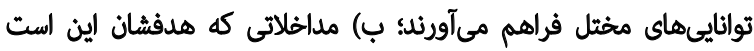

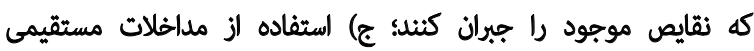

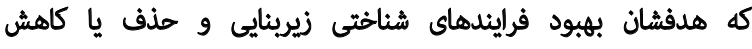

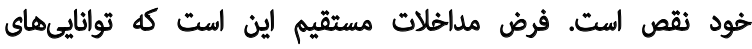

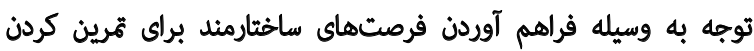

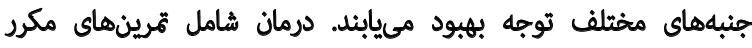

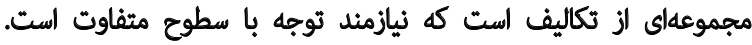

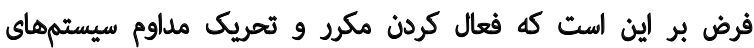

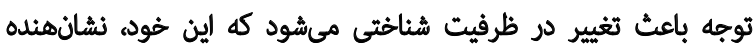

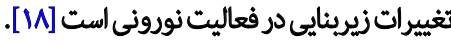

شواهد مثعددى در زمينه اثربخشى بازتوانى شناختى در بهبود نقايص

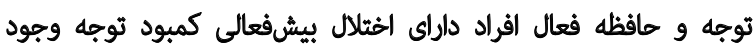

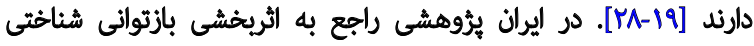

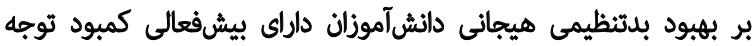

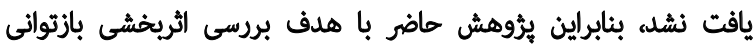

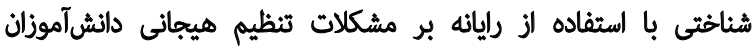

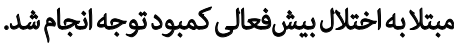

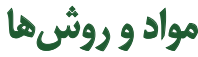

اين يُروهش الز نوع مطالعاث آزمايشى است كه با طرح ييش آزمون

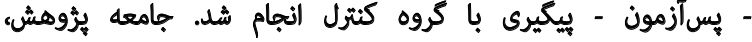

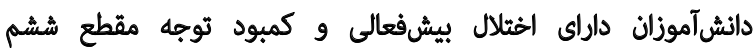
تا نهم منطقه هفت تهران در سال تهصيلى شوالى

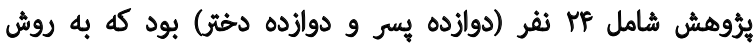

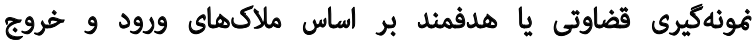

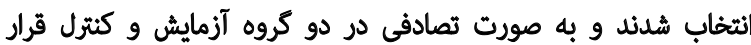

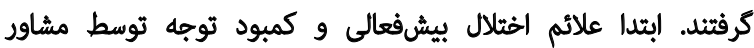

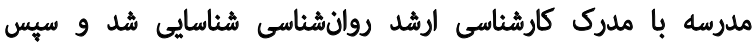

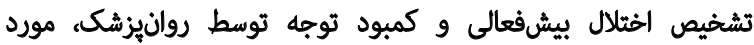

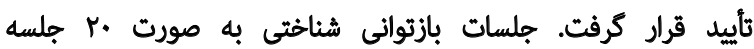

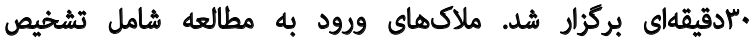

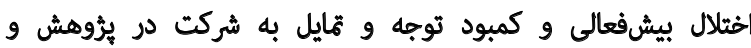

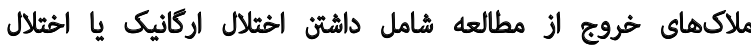

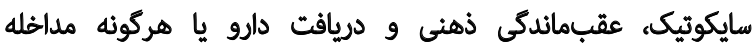

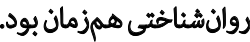

توانايى فرد براي تغيير و اهلاح حالتهاى هيجانى تعريف شده

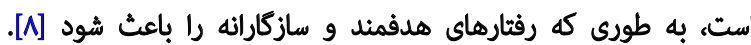

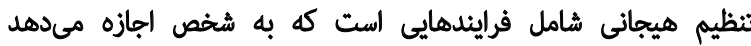

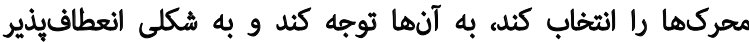

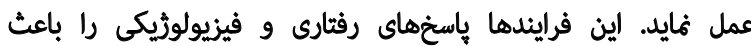

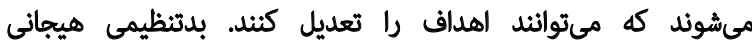
زمانى ايجاد مىشود كه اين فرايندهائ انطباقى منجر به به شكست

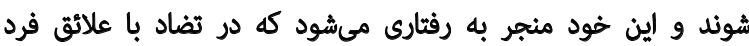

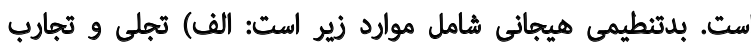

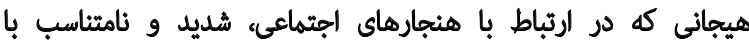

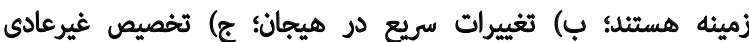
توجه به محركهاي هيجاني.

بدتنظيمى هيجانى يك صفت هاندبعدى است كه منحصر به به

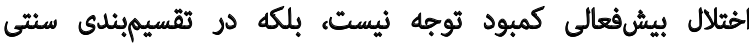

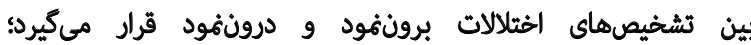

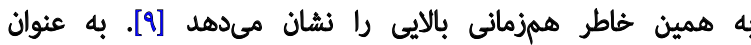

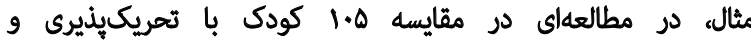
بدتنظيمى هيجانى و اختلال بيشفعالى كمبود توجه و و هوبن كودى

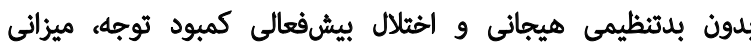

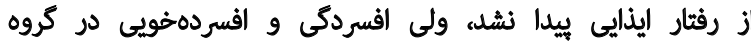

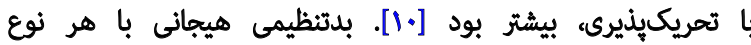

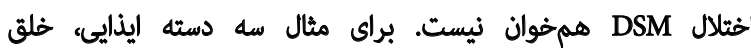

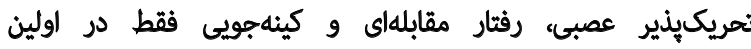

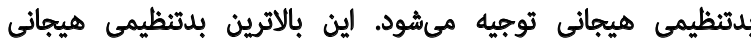

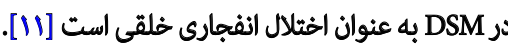

توجه يك عنصر ههم تنظيم هيجانى است [IIT]. سوكيرىهاى

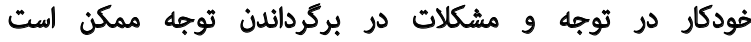

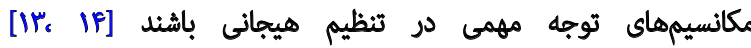

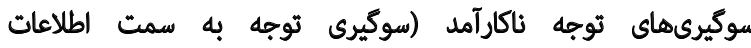

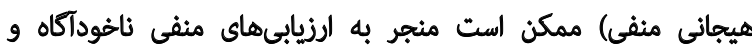

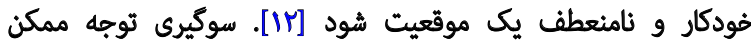

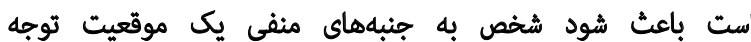

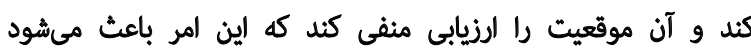

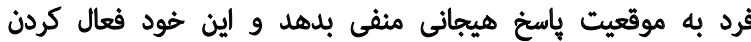

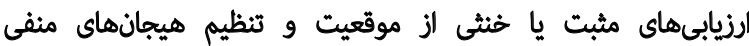

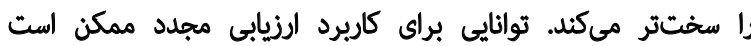

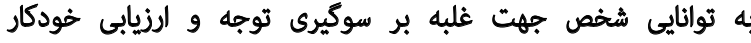

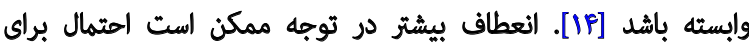

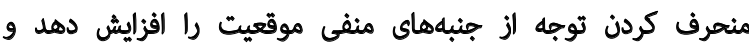

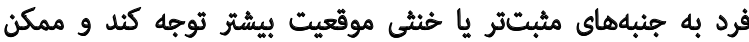

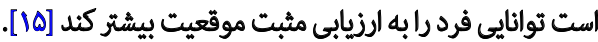
به علت نقش توجه در تنظيم هيجان، آموزش توجه ممكن است

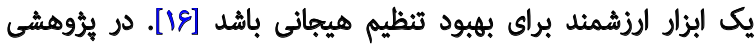


ياستخ دهد يا در هاسخ دادن شكست بخورد بازخوردى به شكل غرش

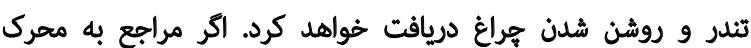
نامربوط ياسخ دهد هراغ قرمز روشن خواهد شد.

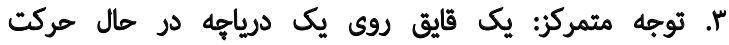

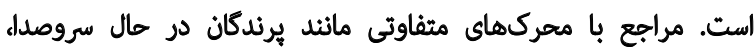

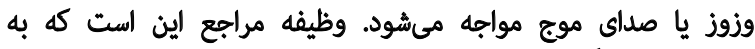

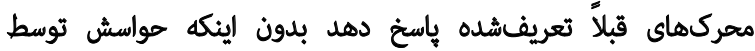

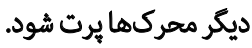

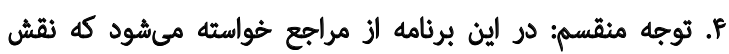

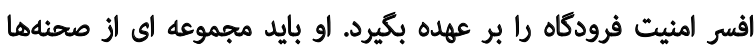

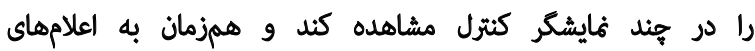

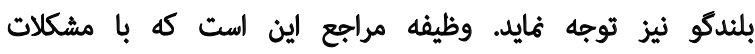

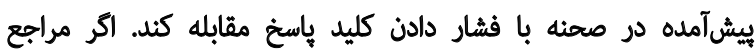

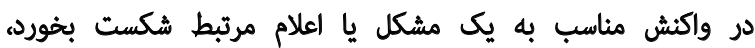

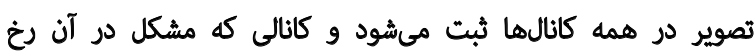

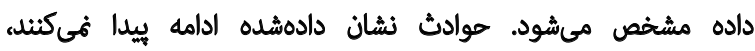

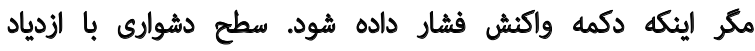
كانالهايي كه بايد يايش شوند، تعيين مىشود.

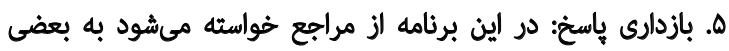

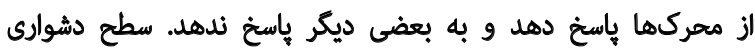

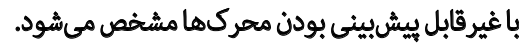

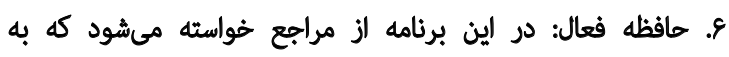

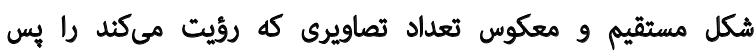

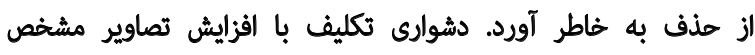

مى شود.

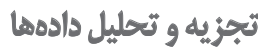

دادههاى اين يزوهش در دو بخش توصيفى (ميانكين و انحراف

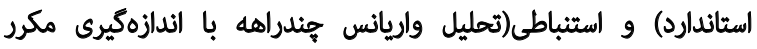

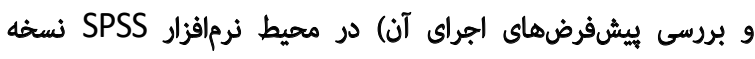

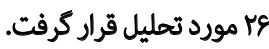

يافتهها

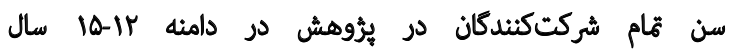

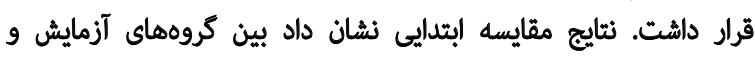

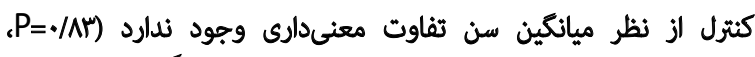

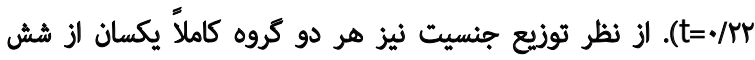

دختر و شش يسر تشكيل شدند (جدول شماره ا).

براى اجراى آزمونهاى آماري، ابتدا بيشفرضهاى آمارى مورد

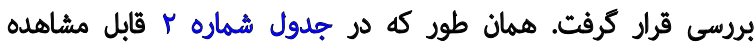

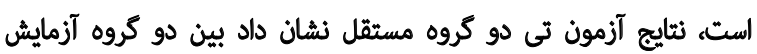

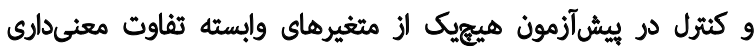

هيرسشامها دشوارى در تنظيم هيجانى (فرم اصلاحشده برايى

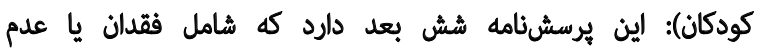

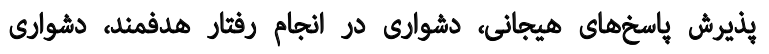

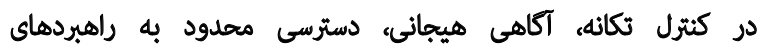

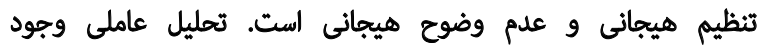

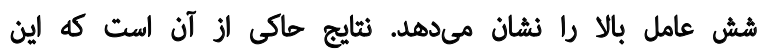

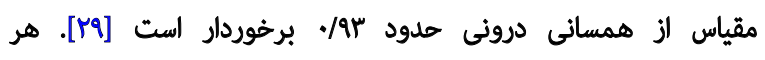

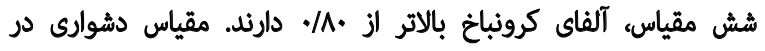

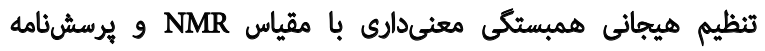

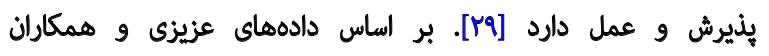

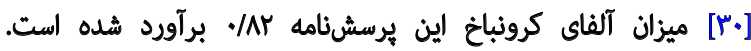

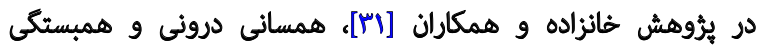

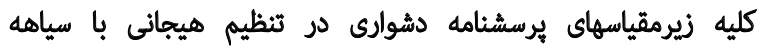

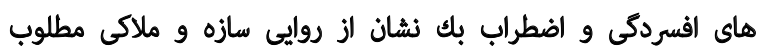

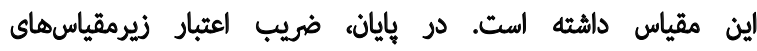

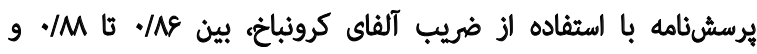

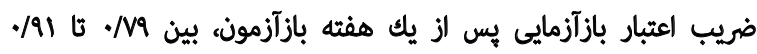

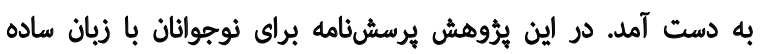

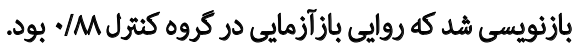

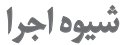

بروتكل: يروتكل مداخله بازتوانى شناختى با استفاده از رايانه به توزيه

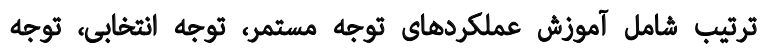

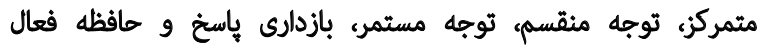

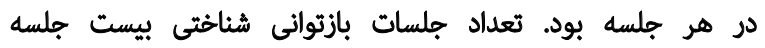

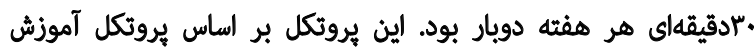

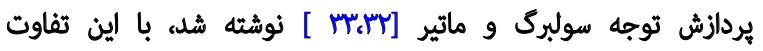

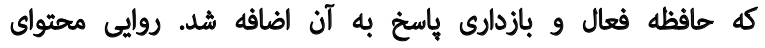

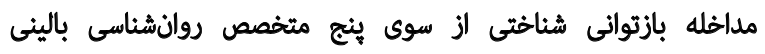
وروانيزشك مورد ثأييد قرار كرفت.

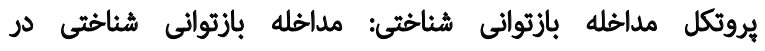

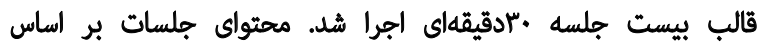

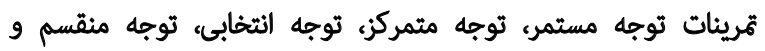

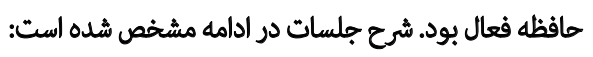

1. توجه مستمر: يك موتورسيكلت در جاده حركت مىكند. وظيفه

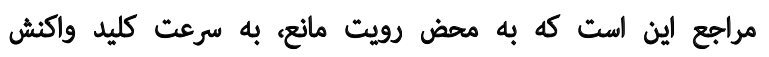
را فشار دهد. سطح دشوارى با كاهش زمان واكنش تعيين مىشود.

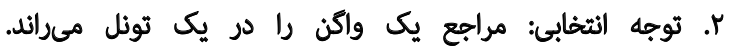

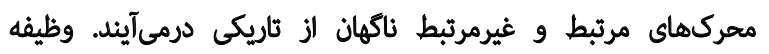

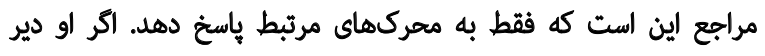




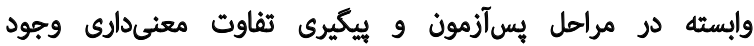

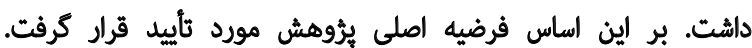

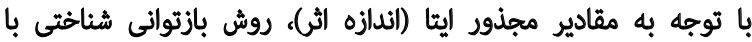

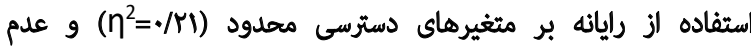

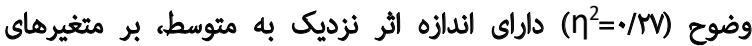

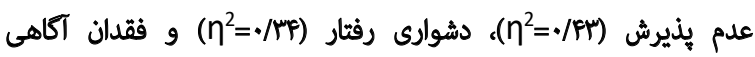
(اراي اندازه اثر متوسط و بر مثئيرهاى كنترل ثكانه

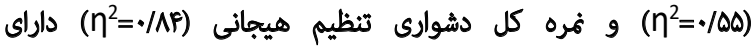

اندازه اثر بالا بود (جدول شماره").

بحث

در مطالعه حاضر، اثربخشى بازتوانى شناختى با استفاده از رايانه

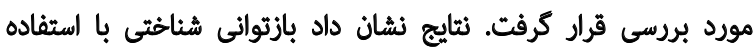

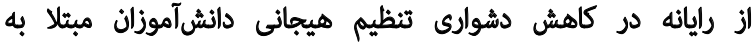

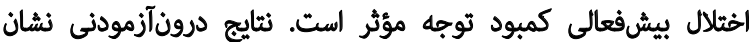

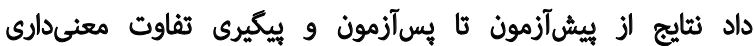

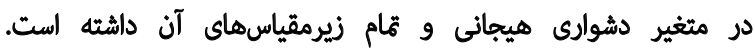

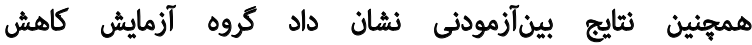

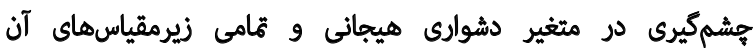

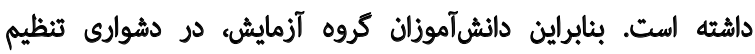

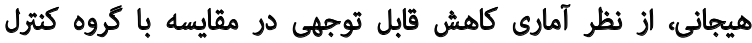

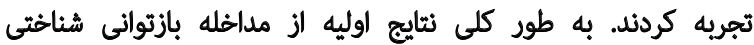
به عنوان يك مداخله اثربخش برائ بهائ بهبود دشوارى تنظيم هيجانى
وجود ندارد؛ اين يافتهها نشان ميدهد كه تخصيص تصادفى به صورت

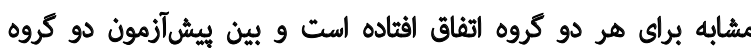

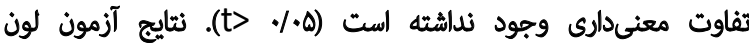

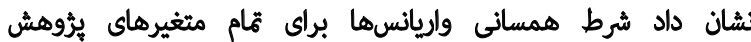

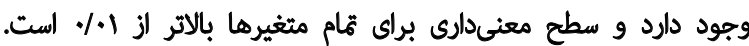

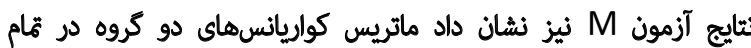

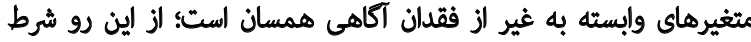

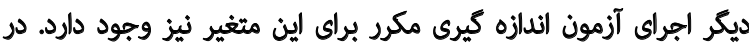

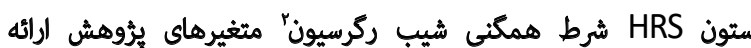

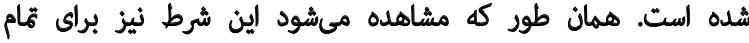

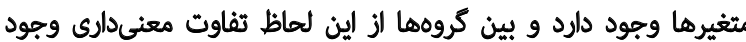

ندارد (جدول شمارهr).

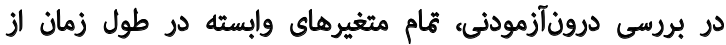

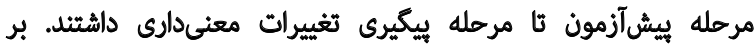

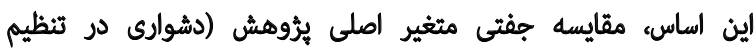

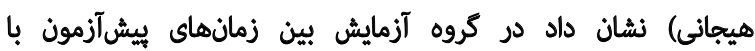

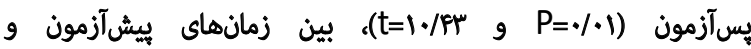

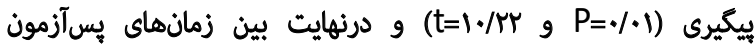

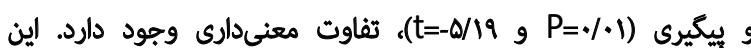

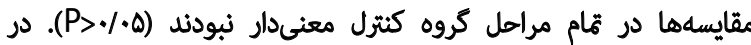

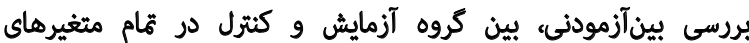

2. Homogeneity of Regression Slopes (HRS)

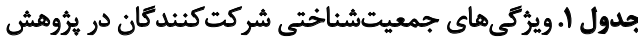

\begin{tabular}{|c|c|c|c|c|c|}
\hline \multirow{2}{*}{ معثى سطارى } & \multirow{2}{*}{ t مقدار t } & \multicolumn{3}{|c|}{ ميانكين +|نحراف معيار } & \multirow{2}{*}{ ويزّكى } \\
\hline & & كل & أزمايش & كتترل & \\
\hline TI &.$/ M T$ & Ir/EY./AY & $1 \% / \Delta A \pm \cdot / 9$. & $\mid r / e V \pm \cdot / M$ & سن (سال) \\
\hline- & - & ir & 8 & $\varepsilon$ & جنسيت يسر (تعلاد) \\
\hline - & - & ir & 8 & $\&$ & جنسيت دختر (تعلداد) \\
\hline
\end{tabular}

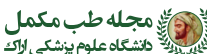

جدول ז. آزمون برابرى واريانسها ومقايسه ميائكينهاي بيش آزمون متغيرهاي مورد اندازهكيرى دو كروه آزمايش و كنترل

\begin{tabular}{|c|c|c|c|c|c|c|c|c|c|c|c|}
\hline $\mathbf{P}$ & HRS & $\mathbf{P}$ & L-E-V & $\mathbf{P}$ & $\mathbf{F}$ & Box's M & $\mathbf{P}$ & $\mathbf{t}$ & $\mathbf{P}$ & L-V & هتغير \\
\hline / Rr & $\cdot / \Delta F^{F}$ &.$/ N T$ & $T / M T$ & .119 & 1/ee & $1 \% / 18$ & .198 &.$/ 18$ &.$/ 19$ & $\mid / A 1$ & عدم يذيرش \\
\hline.$/ 0$ & 1.119 & 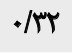 & ITE & $+1+r$ & $1 / A 1$ & $F \Delta / F q$ &.$/ 98$ & .1 .8 & - /va &.$/ 11$ & دشوارى رفتار \\
\hline . & ./9T &.$/ 1 \%$ & r/Ir &.$/ \pi T$ & $1 / R \Delta$ & $T / / N$ &.$/ R A$ & I/I & $\cdot / N^{*}$ & ./N & كثترل تكانه \\
\hline . IAV & 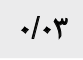 &.$/ r$ & Pleq & .1 .1 & $r / \bullet A$ & $\Delta r / 19$ &.$/ M$ & .118 &.$/ R A$ & - $/ \Delta T$ & فقعدان أكاهي \\
\hline.$/ 1 V$ & $5 / .9$ &.$/ T V$ & Ver &.$/ N T$ & $1 / f^{+1}$ & $r \Delta / r^{n}$ & . /Ar &.$/ M T$ &.$/ 2 \mathrm{~V}$ &.$(\Delta)^{f}$ & دسترسي هحدود \\
\hline.$/ 94$ & .1 .1 &.$/ 99$ & $\cdot / \cdot r$ &.$/ r$. & $1 / m e$ & $M / q$. &.$/ F T$ & ./AT & .180 & 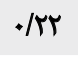 & عدم وضوح \\
\hline.$/ M$ & $.1 . r$ & $1+r$ & me. &..$r$ & $M$ & $p r / p q$ & .11 & $I / M^{2}$ &.$/ \& A$ & - $/ A T$ & كل \\
\hline
\end{tabular}


جدول ب. ميانكين و انحراف استاندارد متغيرهاى يُروهش و نتايج تحليل واريانس جندراهه با اندازهيرى مكرر در مراحل ييش آزمون، يس آزمون و يبيكيرى

\begin{tabular}{|c|c|c|c|c|c|c|c|c|c|}
\hline \multicolumn{3}{|c|}{ بين أزمودنى } & \multicolumn{3}{|c|}{ درون أزمودنى } & \multicolumn{2}{|c|}{ مياتكين|نحراف معيار } & \multirow{2}{*}{ مرحله } & \multirow{2}{*}{ مثغير } \\
\hline$\eta^{\gamma}$ & $\mathbf{P}$ & $F$ & $\eta^{r}$ & $P$ & $F$ & آزايش & كثترل & & \\
\hline . & $+1+1$ & $|\Delta| \cdot f$ & $\cdot M^{F}$ & $.1+1$ & $\Delta V / q V$ & 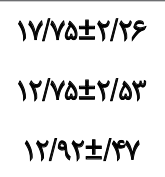 & 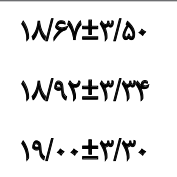 & 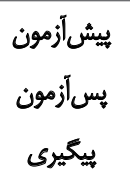 & عدم ئيرش \\
\hline.$/ M F$ & .101 & $1 . / 4 T$ & . $1 \Delta E$ & .1 .1 & $r \Delta / r \Delta$ & $\begin{array}{l}|N| V \pm T / M \mid \\
\mid r / F T \pm T / S \Delta \\
\mid r / * . \pm T / r\end{array}$ & $\begin{array}{l}W \cdot \Lambda \pm r / r r \\
W \cdot \Lambda \pm r / \cdot q \\
W r \Delta \pm r / q r\end{array}$ & 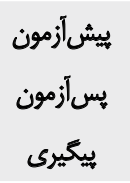 & دشوارى رفتار \\
\hline $.10 \Delta$ & .1 .1 & melle & . IEV & .1 .1 & FI/TA & 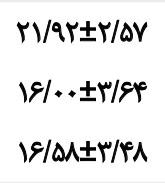 & $\begin{array}{l}r / \cdot \Lambda \pm r / \Delta \cdot \\
r / \Delta \cdot \pm r / \Lambda \Delta \\
r / \& V \pm r / \cdot \varnothing\end{array}$ & 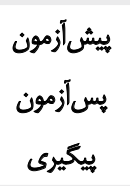 & كتّرل تكاثه \\
\hline . $/ 41$ & .101 & IF/IT & $.10 \mathrm{~A}$ &.$/ 1$ & TWIET & 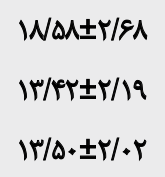 & 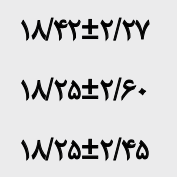 & يسيش آزئزون & فقدان آكاهي \\
\hline.$/ M I$ & $1+r$ & $\Delta / M$ & .109 &.$/ 1$ & rq. 1 & $\begin{array}{l}r / / A \pm r / V \Delta \\
\mid \Delta / \Delta \Lambda \pm r / \cdot r \\
\mid \Delta / \Lambda r \pm r / l .\end{array}$ & $\begin{array}{l}r \cdot / V \Delta \pm \varphi / \Delta \Lambda \\
r \cdot / \Delta \Lambda \pm r / Q Q \\
r \cdot / V \Delta \pm F / / F\end{array}$ & يبيش آزآزمون & دسترسى محدود \\
\hline$\cdot / r V$ & +4 & V/Fa &.$/ 4 A$ & $+1+1$ & WTA & $\begin{array}{l}|\varepsilon /| V \pm \Psi /+r \\
\mid V / q r \pm r /+\varepsilon \\
\mid r / \Delta A \pm r / r r\end{array}$ & $\begin{array}{l}I V / \Delta \cdot \pm r / a f \\
I V / \Delta \cdot \pm r / \Delta \Delta \\
I V / \Delta \cdot \pm r / \Lambda .\end{array}$ & ييش ئيسأزمون & علدم وضوح \\
\hline - /AF & $.1+1$ & $1 \cdot V / M$ & . Ar & $.1+1$ & Q S/D9 & 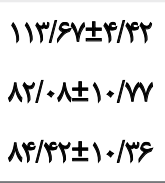 & 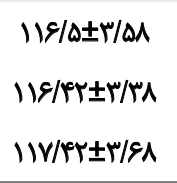 & ييس آلزمون & 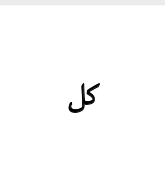 \\
\hline
\end{tabular}

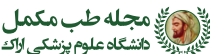

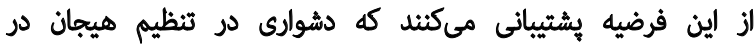

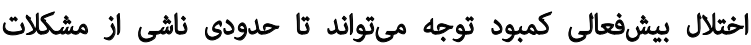

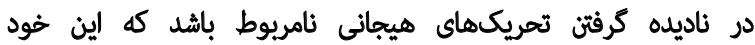

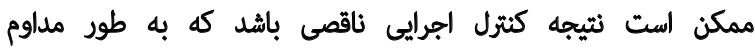

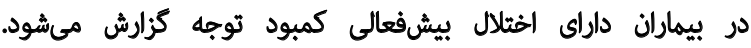

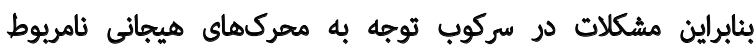

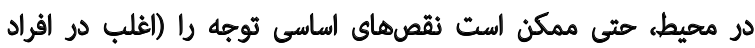

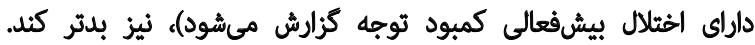

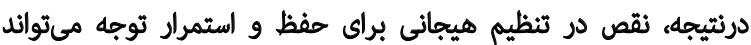

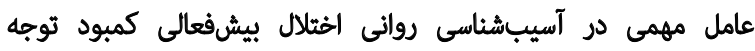

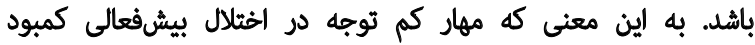

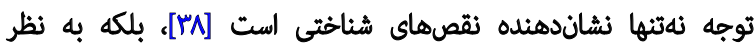
مىرسدتحت شرايط حواسيرتى هيجانى تقويت نيز مى شود. توجه يكى از جنبههاى اصلى ساخثار شناختى است كه در ساختار
دانشآموزان مبثلا به اغتلال بيشفعالى كمبود توجه حمايت

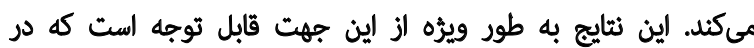

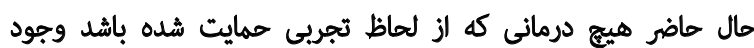

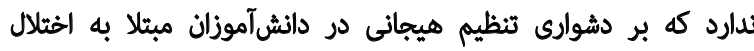
بيشفعالى كمبود توجهاه مركز داشته باشد.

يُروهشهاى زيادى نشاندهثده وجود دشوارى در تنظيم هيجانى

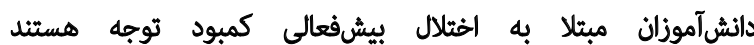

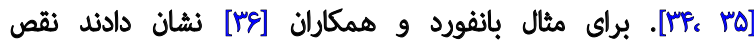

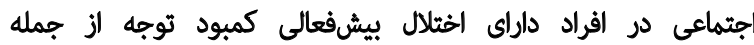

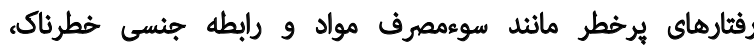

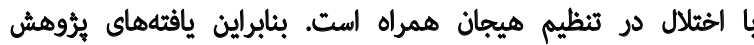

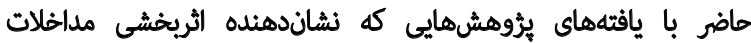

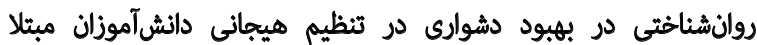

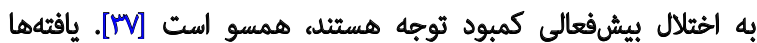


ييامدهاى بالينى: مطالعه كنونى يك كام مهم در بهبود ثاثير مخرب

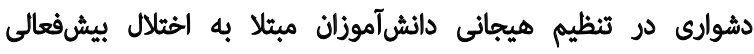

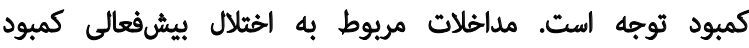

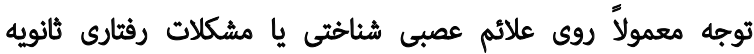

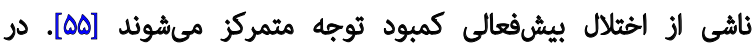

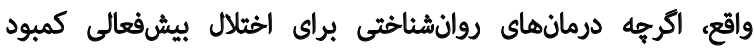

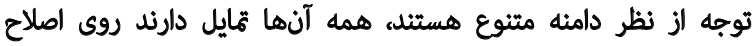

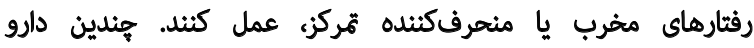

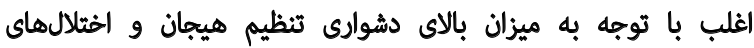

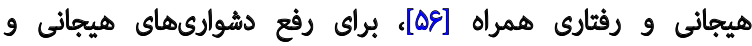

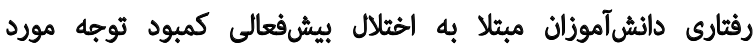

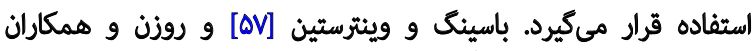

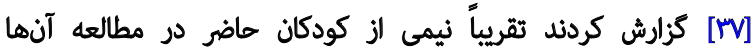

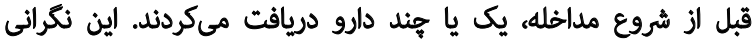

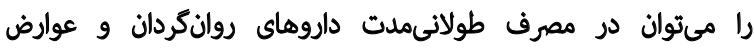

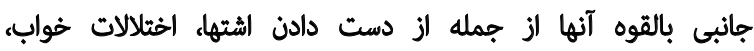

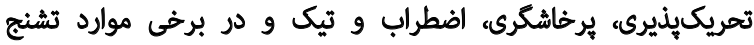

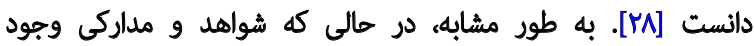

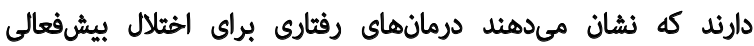

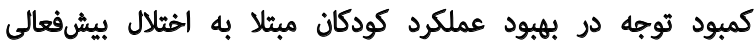

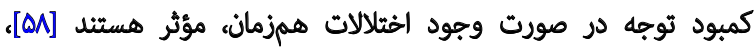

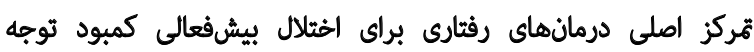

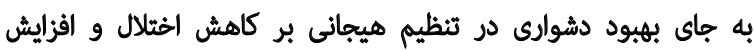

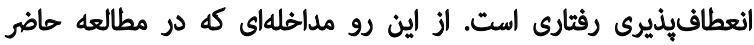

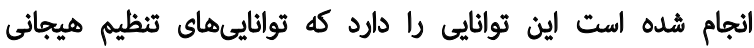

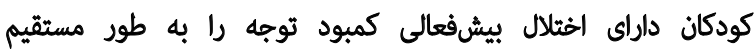

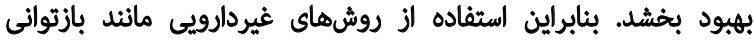

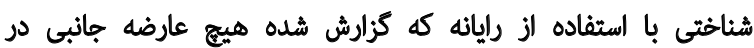

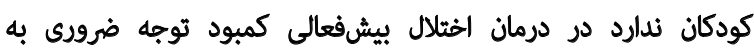

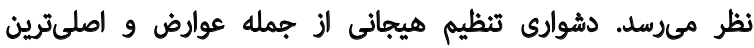

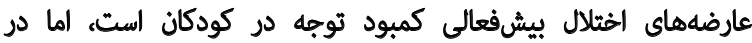

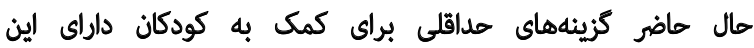

اخثلال وجود دارد [بر]

\section{نتيجليكيرى}

مداخله بازتوانى شناختى با استفاده از رايانه كه در مطالعه حاضر

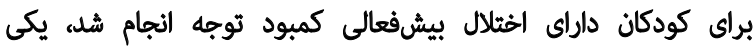

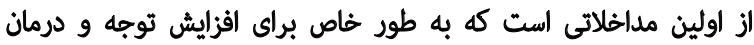

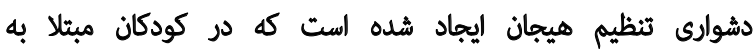

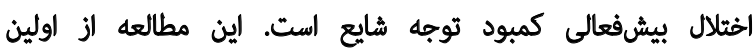

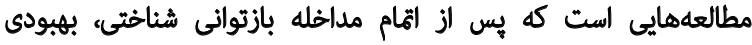

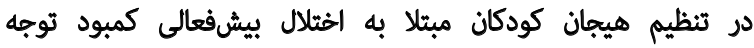

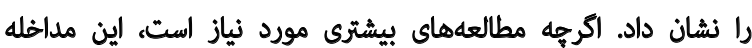

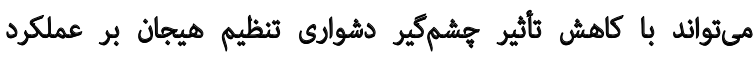

هوش، حافظه و ادراك نيز نقش مهمى دارد. نشان داده شده است

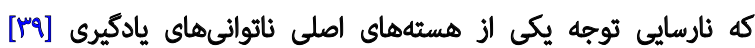

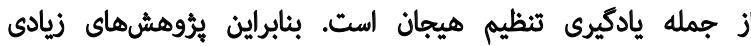

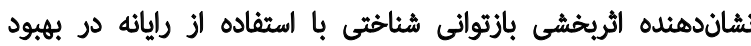

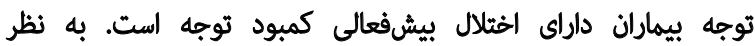

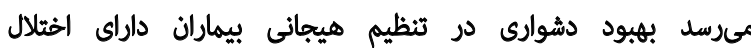

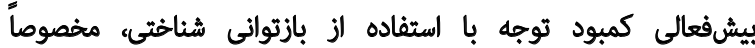
متغير توجه، با توجه به مشكلات توجه در اين بيهاران قابل فهم باشد.

نتايج بهدستآمده از ئزوهش حاضر با نثايج ئزوهشهاى زيادى

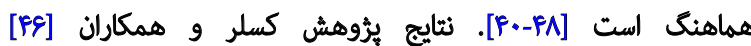

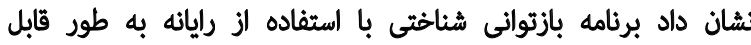

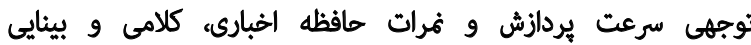

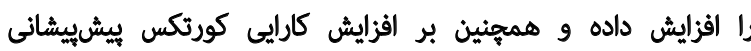

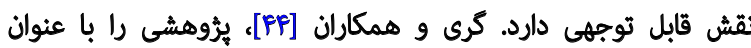

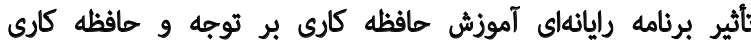

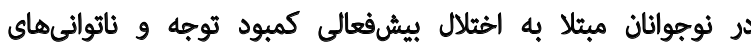

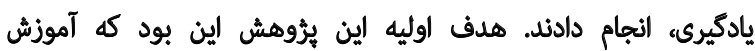

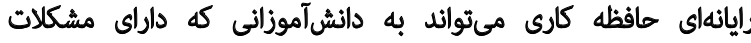

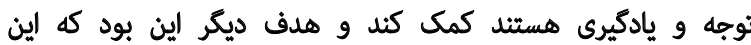

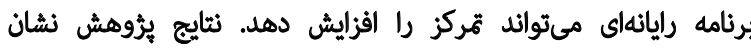

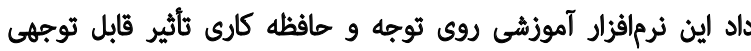

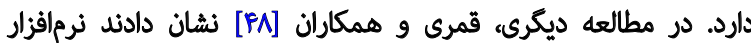

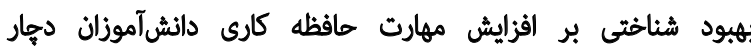

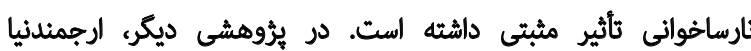

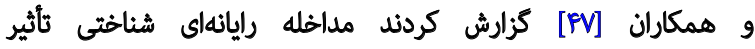

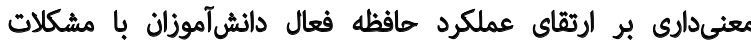

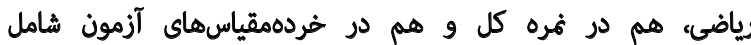
مافظاه، مازها، مكعب هائ رو به جلو $و$ رو به عقب داشته الست. هرن.

مطالعات اخير با استفاده از تصويريردارى رزونانس مغناطيسى

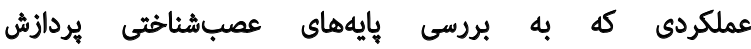

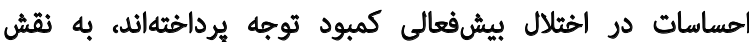

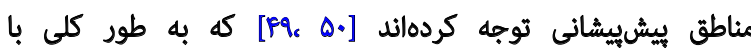

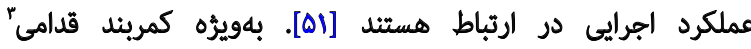

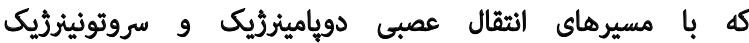

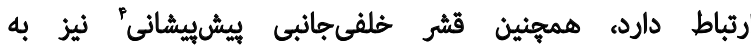

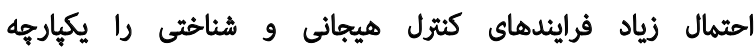

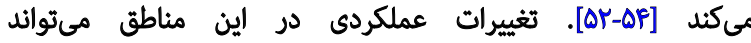

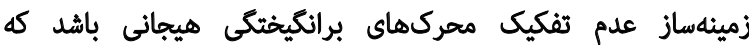

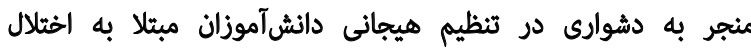

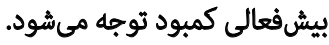

3. Anterior cingulate

4. Dorsolateral prefrontal cortex 


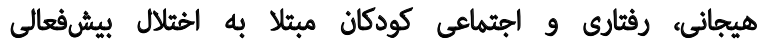

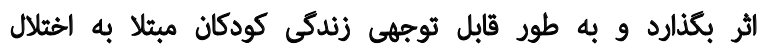

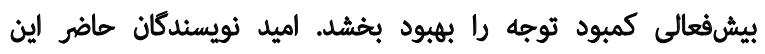

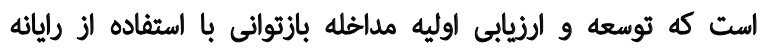

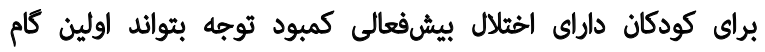

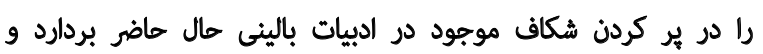
باعث بهبود و اثربخشى معنىدار و بالينى در عملكرد كودكان مبثلا

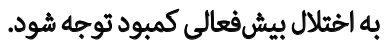

اين مطالعه داراى محدوديتهايى نيز است. در ابتدا اين مطالعه

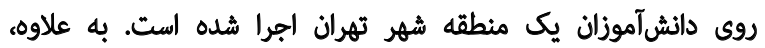

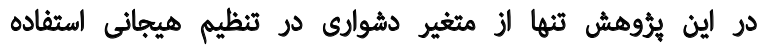

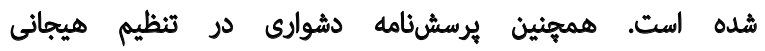

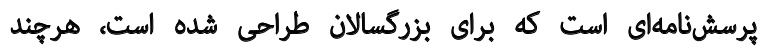

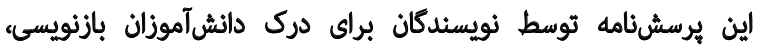

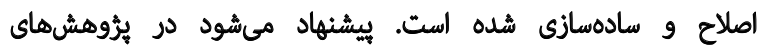

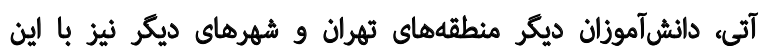

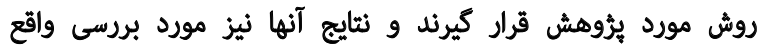

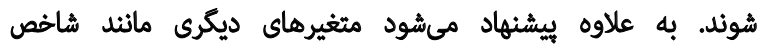

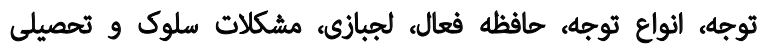

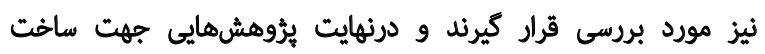

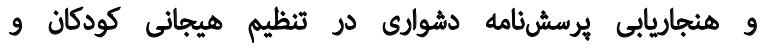
نوجوانان اجرا شوند.

مالافظات الخاقي

\section{ييروى از اصول اخالاق يثوهش}

كدهاى اخلاقى APA ، IRPCO نظر كرفته شيد.

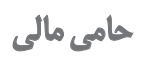
اين مقاله از رساله دكترى نويسئده اوله در كروه روانشناسي،

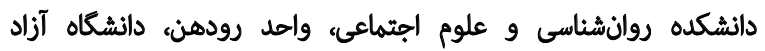
اسلامى استخر اج شده استيت. مشاركتونتويسنان تمام نويسندكان در آمادهازي اين مقاله مشاركت داشتّاند.

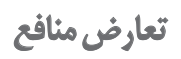
نويسندكان مقاله هيجكونه تعارضى در مناقع اعلام نكردند. 


\section{References}

[1] Sadock BJ, Sadock VA, Ruiz P. Kaplan \& Sadock's synopsis of psychiatry: Behavioral sciences/clinical psychiatry $11^{\text {th }}$ ed. Netherlands: Wolters Kluwer; 2015. https://books.google.com/books/about/Kaplan_Sadock_s_ Synopsis_of_Psychiatry.html?id=QQmOngEACAA

[2] Jensen SA, Rosén LA. Emotional reactivity in children with attentiondeficit/hyperactivity disorder. Journal of Attention Disorders. 2004; 8(2):53-61. [DOI:10.1177/108705470400800203] [PMID]

[3] Braaten EB, Rosén LA. Self-regulation of affect in attention deficit-hyperactivity disorder (ADHD) and non-ADHD boys: Differences in empathic responding. Journal of Consulting and Clinical Psychology. 2000; 68(2):313-21. [DOI:10.1037/0022-006X.68.2.313] [PMID]

[4] Factor PI, Rosen PJ, Reyes RA. The relation of poor emotional awareness and externalizing behavior among children with ADHD. Journal of Attention Disorders. 2016; 20(2):168-77. [DOI:10.1177/1087054713494005] [PMID]

[5] Clements SD. Minimal brain dysfunction in children: Terminology and identification: Phase one of a three-phase project. Washington, DC: US Department of Health, Education, and Welfare; 1966. https://books. google.com/books/about/Minimal_Brain Dysfunction in Children. $\mathrm{html}$ ?id=AbpsAAAAMAAJ

[6] Shaw P, Stringaris A, Nigg J, Leibenluft E. Emotion dysregulation in attention deficit hyperactivity disorder. The American Journal of Psychiatry. 2014; 171(3):276-93. [DOI:10.1176/appi.ajp.2013.13070966] [PMID] [PMCID]

[7] Bunford N, Dawson AE, Evans SW, Ray AR, Langberg JM, Owens JS, et al. The difficulties in emotion regulation scale-parent report: A psychometric investigation examining adolescents with and without ADHD. Assessment. 2020; 27(5):921-40. [DOI:10.1177/1073191118792307] [PMID]

[8] Thompson RA. Emotion regulation: A theme in search of definition. Monographs of the Society for Research in Child Development. 1994; 59(2-3):25-52. [DOI:10.1111/j.1540-5834.1994.tb01276.x] [PMID]

[9] Achenbach TM, Rescorla LA. Manual for the ASEBA school-age forms \& profiles. Burlington, VT: University of Vermont, Research Center for Children, Youth, \& Families; 2001. https://www.amazon.com/ManualASEBA-School-Age-Forms-Profiles/dp/0938565737

[10] Ambrosini PJ, Bennett DS, Elia J. Attention deficit hyperactivity disorder characteristics: Il. Clinical correlates of irritable mood. Journal of Affective Disorders. 2013; 145(1):70-6. [DOI:10.1016/j.jad.2012.07.014] [PMID] [PMCID]

[11] Abikoff HB, Jensen PS, Arnold LLE, Hoza B, Hechtman L, Pollack S, et al. Observed classroom behavior of children with ADHD: Relationship to gender and comorbidity. Journal of Abnormal Child Psychology. 2002; 30(4):349-59. [DOI:10.1023/A:1015713807297] [PMID]

[12] Wadlinger HA, Isaacowitz DM. Fixing our focus: Training attention to regulate emotion. Personality and Social Psychology Review. 2011; 15(1):75-102. [DOI:10.1177/1088868310365565] [PMID] [PMCID]

[13] Joormann J, Gotlib IH. Emotion regulation in depression: Relation to cognitive inhibition. Cognition \& Emotion. 2010; 24(2):281-98. [DOI:10.1080/02699930903407948] [PMID] [PMCID]

[14] Joormann J, D’Avanzato C. Emotion regulation in depression: Examining the role of cognitive processes: Cognition \& emotion lecture at the 2009 ISRE meeting. Cognition and Emotion. 2010; 24(6):913-39. [DOI:10.1080/02699931003784939]
[15] Siemer M, Reisenzein R. Emotions and appraisals: Can you have one without the other? Emotion. 2007; 7(1):26-9. [DOI:10.1037/15283542.7.1.26] [PMID]

[16] Johnson DR. Goal-directed attentional deployment to emotional faces and individual differences in emotional regulation. Journal of Research in Personality. 2009; 43(1):8-13. [DOI:10.1016/j.jrp.2008.09.006]

[17] van Tilburg WAP, Igou ER. Boredom begs to differ: Differentiation from other negative emotions. Emotion. 2017; 17(2):309-22. [DOI:10.1037/ emo0000233] [PMID]

[18] Mateer CA, Kerns KA, Eso KL. Management of attention and memory disorders following traumatic brain injury. Journal of Learning Disabilities. 1996; 29(6):618-32. [DOI:10.1177/002221949602900606] [PMID]

[19] Rapoport JL, Gogtay N. Brain neuroplasticity in healthy, hyperactive and psychotic children: Insights from neuroimaging. Neuropsychopharmacology. 2008; 33(1):181-97. [DOI:10.1038/sj.npp.1301553] [PMID]

[20] Olesen PJ, Westerberg H, Klingberg T. Increased prefrontal and parieta activity after training of working memory. Nature Neuroscience. 2004 7(1):75-9. [DOI:10.1038/nn1165] [PMID]

[21] Castellanos FX, Giedd JN, Berquin PC, Walter JM, Sharp W, Tran T, et al. Quantitative brain magnetic resonance imaging in girls with attention-deficit/hyperactivity disorder. Archives of General Psychiatry. 2001 58(3):289-95. [DOI:10.1001/archpsyc.58.3.289] [PMID]

[22] Filipek PA, Semrud-Clikeman M, Steingard RJ, Renshaw PF, Kennedy DN, Biederman J. Volumetric MRI analysis comparing subjects having attention-deficit hyperactivity disorder with normal controls. Neurology. 1997; 48(3):589-601. [DOI:10.1212/WNL.48.3.589] [PMID]

[23] Klingberg T, Fernell E, Olesen PJ, Johnson M, Gustafsson P, Dahlström K et al. Computerized training of working memory in children with ADHD-a randomized, controlled trial. Journal of the American Academy of Child \& Adolescent Psychiatry. 2005; 44(2):177-86. [DOI:10.1097/00004583200502000-00010] [PMID]

[24] Kerns KA, Eso K, Thomson J. Investigation of a direct intervention for improving attention in young children with ADHD. Developmental Neuropsychology. 1999; 16(2):273-95. [DOI:10.1207/S15326942DN1602_9]

[25] Bikic A, Leckman JF, Lindschou J, Christensen T $\varnothing$, Dalsgaard S. Cognitive computer training in children with Attention Deficit Hyperactivity Disorder (ADHD) versus no intervention: Study protocol for a randomized controlled trial. Trials. 2015; 16:480. [DOI:10.1186/s13063-015-0975-8] [PMID] [PMCID]

[26] Rabiner DL, Murray DW, Skinner AT, Malone PS. A Randomized Trial of Two Promising Computer-Based Interventions for Students with Attention Difficulties. Journal of Abnormal Child Psychology. 2010; 38(1):131 42. [DOI:10.1007/s10802-009-9353-x] [PMID]

[27] Robatmili S. The effect of computer-assisted cognitive rehabilitation on working memory in children with ADHD. International Journal of Psychology (IPA). 2019; 13(1):183-205. [DOI:10.24200/ijpb.2018.115071.]

[28] Emadian SO, Bahrami H, Hassanzadeh R, Bani-jamali S. Effects of narrative therapy and computer-assisted cognitive rehabilitation on the reduction of ADHD symptoms in children. Journal of Babol University Of Medical Sciences. 2016; 18(6):28-34. [DOI: 10.22088/jbums.18.6.28]

[29] Gratz KL, Roemer L. Multidimensional assessment of emotion regulation and dysregulation: Development, factor structure, and initial validation of the difficulties in emotion regulation scale. Journal of Psychopathology and Behavioral Assessment. 2004; 26(1):41-54 [DOI:10.1023/B:JOBA.0000007455.08539.94] 
[30] Azizi A, Mirzaei A, Shams J. [Correlation between distress tolerance and emotional regulation with students smoking dependence (Persian)]. Hakim Research Journal. 2010; 13(1):11-8. http://hakim.hbi. ir/article-1-608-en.html

[31] Khanzadeh M, Saeediyan M, Hosseinchari M, Edrissi F. [Factor structure and psychometric properties of difficulties in emotional regulation scale (Persian)]. International Journal of Behavioral Sciences. 2012; 6(1):87-96. http://www.behavsci.ir/article 67768.htm

[32] Sohlberg $M$, Mateer $C$. Attention process training: A program for cognitive rehabilitation to address persons with attentional deficits ranging from mild to severe. $3^{\text {th }}$ ed. Wake Forest, North Carolina: Lash \& Associates Publishing; 2005.

[33] Thomson J, Kerns K, Seidenstrang L, Sohlberg MM, Mateer CA. Pay attention: A children's attention process training program. Wake Forest, North Carolina: Lash \& Associates Publishing; 2005

[34] Maedgen JW, Carlson CL. Social functioning and emotional regulation in the attention deficit hyperactivity disorder subtypes. Journal of Clinical Child Psychology. 2000; 29(1):30-42. [DOI:10.1207/ S15374424jccp2901_4] [PMID]

[35] Walcott CM, Landau S. The relation between disinhibition and emotion regulation in boys with attention deficit hyperactivity disorder. Journal of Clinical Child and Adolescent Psychology. 2004; 33(4):772-82. [DOI:10.1207/s15374424jccp3304_12] [PMID]

[36] Bunford N, Evans SW, Wymbs F. ADHD and emotion dysregulation among children and adolescents. Clinical Child and Family Psychology Review. 2015; 18(3):185-217. [DOI:10.1007/s10567-015-0187-5] [PMID]

[37] Rosen PJ, Leaberry KD, Slaughter K, Fogleman ND, Walerius DM, Loren REA, et al. Managing Frustration for Children (MFC) group intervention for ADHD: An open trial of a novel group intervention for deficient emotion regulation. Cognitive and Behavioral Practice. 2019; 26(3):522-34. [DOI:10.1016/j.cbpra.2018.04.002]

[38] Schwartz K, Verhaeghen P. ADHD and Stroop interference from age 9 to age 41 years: A meta-analysis of developmental effects. Psychological Medicine. 2008; 38(11):1607-16. [DOI:10.1017/ S003329170700267X] [PMID]

[39] Şahin B, Karabekiroğlu K, Bozkurt A, Usta MB, Aydın M, Çobanoğlu $C$. The relationship of clinical symptoms with social cognition in children diagnosed with attention deficit hyperactivity disorder, specific learning disorder or autism spectrum disorder. Psychiatry Investigation. 2018; 15(12):1144-53. [DOI:10.30773/pi.2018.10.01] [PMID] [PMCID]

[40] Tajik-Parvinchi D, Wright L, Schachar R. Cognitive rehabilitation for Attention Deficit/Hyperactivity Disorder (ADHD): Promises and problems. Journal of the Canadian Academy of Child and Adolescent Psychiatry. 2014; 23(3):207-17. [PMID] [PMCID]

[41] Beck SJ, Hanson CA, Puffenberger SS, Benninger KL, Benninger WB. A controlled trial of working memory training for children and adolescents with ADHD. Journal of Clinical Child and Adolescent Psychology. 2010; 39(6):825-36. [DOI:10.1080/15374416.2010.517162 ] [PMID]

[42] Chacko A, Bedard AC, Marks DJ, Feirsen N, Uderman JZ, Chimiklis $A$, et al. A randomized clinical trial of Cogmed Working Memory Training in school-age children with ADHD: A replication in a diverse sample using a control condition. Journal of Child Psychology and Psychiatry, and Allied Disciplines. 2014; 55(3):247-55. [DOI:10.1111/ jcpp.12146] [PMID] [PMCID]
[43] Gibson BS, Gondoli DM, Johnson AC, Steeger CM, Dobrzenski BA Morrissey RA. Component analysis of verbal versus spatial working memory training in adolescents with ADHD: A randomized, controlled trial. Child Neuropsychology. 2011; 17(6):546-63. [DOI:10.10 80/09297049.2010.551186] [PMID]

[44] Gray SA, Chaban P, Martinussen R, Goldberg R, Gotlieb H, Kronitz R, et al. Effects of a computerized working memory training program on working memory, attention, and academics in adolescents with severe LD and comorbid ADHD: A randomized controlled trial. Journal of Child Psychology and Psychiatry. 2012; 53(12):1277-84. [DOI:10.1111/j.14697610.2012.02592.x] [PMID]

[45] Cortese S, Ferrin M, Brandeis D, Buitelaar J, Daley D, Dittmann RW, et al. Cognitive training for attention-deficit/hyperactivity disorder: Metaanalysis of clinical and neuropsychological outcomes from randomized controlled trials. Journal of the American Academy of Child \& Adolescent Psychiatry. 2015; 54(3):164-74. [DOI:10.1016/j.jaac.2014.12.010] [PMID] [PMCID]

[46] Kesler SR, Lacayo NJ, Jo B. A pilot study of an online cognitive rehabilitation program for executive function skills in children with cancerrelated brain injury. Brain Injury. 2011; 25(1):101-12. [DOI:10.3109/026 99052.2010.536194] [PMID] [PMCID]

[47] Arjmandnia AA, Sharifi A, Rostami R. [The effectiveness of computerized cognitive training on the performance of visual-spatial working memory of students with mathematical problems (Persian)]. Journal of Learning Disabilities. 2014; 3(4):6-24. http://jld.uma.ac.ir/article_186_ en.html

[48] Ghamari Givi H, Narimani M, Mahmoodi H. [The effectiveness of cognition-promoting software on executive functions, response inhibition and working memory of childern with dyslexia and attention dificit/ hyperactivity (Persian)]. Journal of Learning Disabilities. 2012; 1(2):98115. http://jld.uma.ac.ir/article_99_en.html

[49] Passarotti AM, Sweeney JA, Pavuluri MN. Emotion processing influences working memory circuits in pediatric bipolar disorder and attention-deficit/hyperactivity disorder. Journal of the American Academy of Child and Adolescent Psychiatry. 2010; 49(10):1064-80. [DOI:10.1016/j. jaac.2010.07.009] [PMID] [PMCID]

[50] Passarotti AM, Sweeney JA, Pavuluri MN. Differential engagement of cognitive and affective neural systems in pediatric bipolar disorder and attention deficit hyperactivity disorder. Journal of the International Neuropsychological Society. 2010; 16(1):106-17. [DOI:10.1017/ S1355617709991019] [PMID] [PMCID]

[51] Nigg JT. Is ADHD a disinhibitory disorder?. Psychological Bulletin. 2001 127(5):571-98. [DOI:10.1037/0033-2909.127.5.571] [PMID]

[52] Holroyd CB, Coles MGH. The neural basis of human error processing: Reinforcement learning, dopamine, and the error-related negativity. Psychological Review. 2002; 109(4):679-709. [DOI:10.1037/0033295X.109.4.679] [PMID]

[53] Ochsner KN, Ray RD, Cooper JC, Robertson ER, Chopra S, Gabrieli JDE, et al. For better or for worse: Neural systems supporting the cognitive down- and up-regulation of negative emotion. Neurolmage. 2004; 23(2):483-99. [DOI:10.1016/j.neuroimage.2004.06.030] [PMID]

[54] Schulze L, Domes G, Krüger A, Berger C, Fleischer M, Prehn K, et al. Neuronal correlates of cognitive reappraisal in borderline patients with affective instability. Biological Psychiatry. 2011; 69(6):564-73. [DOI:10.1016/j.biopsych.2010.10.025] [PMID]

[55] Watson SMR, Richels C, Michalek AP, Raymer A. Psychosocial treatments for ADHD: A systematic appraisal of the evidence. Journal of Attention Disorders. 2015; 19(1):3-10. [DOI:10.1177/1087054712447857] [PMID] 
[56] Joseph A, Davis K, Fridman M, Gustafsson P, Quintero J, Sikirica V, et al. Characteristics and treatment patterns of children and adolescents with attention-deficit/hyperactivity disorder in real-world practice settings. European Psychiatry. 2016; 33(Suppl 1):S227. [DOI:10.1016/j. eurpsy.2016.01.561]

[57] Bussing R, Winterstein AG. Polypharmacy in attention deficit hyperactivity disorder treatment: Current status, challenges and next steps. Current Psychiatry Reports. 2012; 14(5):447-9. [DOI:10.1007/s11920012-0295-6] [PMID]

[58] Pelham Jr WE, Fabiano GA. Evidence-based psychosocial treatments for attention-deficit/hyperactivity disorder. Journal of Clinical Child and Adolescent Psychology. 2008; 37(1):184-214. [DOI:10.1080/15374410701818681] [PMID] 
This Page Intentionally Left Blank 